\title{
Evaluation of Microal buminuria and Serum Tnf A In Patients with Sepsis and Their Correlation with Disease Severity
}

\author{
Dr. Soumya Knati Dutta ${ }^{1}$, Dr. Sulagna Sahu ${ }^{2}$, Dr. P. K.Thatoi ${ }^{3}$, \\ Dr. Abhay Nath Chaturvedi ${ }^{4}$, Dr. Sanjay Sarkar ${ }^{5}$, Dr. Tushar Kanti Saha ${ }^{6}$ \\ ${ }^{I}$ (Senior Resident, Dept. of Cardiology, Bankura Sammilani Medical College \& Hospital, Bankura); ${ }^{2}(P G T)$, \\ ${ }^{3}$ (Asst. Prof)\} Dept. of Medicine, SCBMCH, Cuttack; $\left\{^{4}(P G T),{ }^{5}(\right.$ RMO cum clinical Tutor $\left.)\right\}$ Dept. of Medicine, \\ Bankura Sammilani Medical College \& Hospital, Bankura, ${ }^{6}$ (Asst. Prof., Dept. of Community Medicine, NRS \\ Medical College, Kolkata)
}

\begin{abstract}
Introduction: Diffuse endothelial dysfunction in sepsis leads to an increase in systemic capillary permeability, the renal component manifesting as micro albuminuria. The degree of microalbuminuria correlates with the severity of acute insult, the quantification of which may serve to predict sepsis and mortality in critically ill patients. Objectives: 1 . To evaluate the degree of microalbuminuria and correlate with severity of sepsis. 2. To evaluate levels of TNFaand severity of sepsis. 3. To correlate degree of microalbuminuria with levels of TNF $\alpha$ in patients with sepsis and its outcome. Methodology: The present study is prospective non interventional study. Patients admitted to medicine ward and ICU staying more than 24 hrs at S.C.B medical college, Cuttack are included in the study, during period of Sept'2011 - Sept'2012. 1. Urine samples were collected within 6 hrs of admission and again at 24 hrs. 2. Urinary microalbumin assay by microalbumin kit (pyrogallol red immunobiometric method) 3.Urinary creatinine estimation by creatinine kit (mod jaffe's kinetic method) 4. Urinary Albumin Creatinine Ratio (ACR) 30-300 mg/gm is microalbuminuria. 5. TNF a is estimated in patients with sepsis by ELISA (bioplex). Results: Patients $(n=75)$ were classified into sepsis,severe sepsis,septic shock and MODS according to American College of chest physician society of critical care consensus. ACR in sepsis group was significantly higher than control group $(P=0.0001)$. Among sepsis cases $A C R$ has a positive correlation with APACHE II score and also distinguishes between survivors and nonsurvivors ( $p=0.001)$. ACR could easily distinguish patients of MODS from sepsis $(p=0.03)$ and septic shock from sepsis $(p=0.007)$. Values of TNFa can distinguish survivors from nonsurvivors $(p=0.04)$ and $T N F \alpha$ also distinguishes patients of sepsis from septic shock $(p=0.002)$. Conclusion: Microalbuminuria has a good diagnostic and prognostic significance in sepsis as compared to APACHE II score and TNF $\alpha$.
\end{abstract}

Keywords: Sepsis, Microalbuminuria, TNFa

\section{Introduction}

Sepsis remains a major global healthcare concern owing to high mortality and morbidity despite the advances in medical therapeutics. ${ }^{1,2}$ Targeted therapies probably lose their efficacy due to late administration. Early goal-directed therapy provides significant benefits with respect to outcome in patients with severe sepsis and septic shock ${ }^{3}$. Sepsis is marked by severe host defence response that involves triggering of potent inflammatory cascade which release a plethora of proinflammatory molecules in circulation. ${ }^{4}$ Blood levels of TNF- $\alpha$ are not elevated in individuals with localized infections, they increase in patients with severe sepsis or septic shock. ${ }^{5}$ TNF- $\alpha$ upregulates expression of arginase in endothelial cells, which leads to O2(-) production then induces endothelial dysfunction. ${ }^{6}$ Diffuse endothelial dysfunction in sepsis leads to an increase in systemic capillary permeability, the renal component manifesting as micro-albuminuria. The degree of micro albuminuria correlates with severity of acute insult. The quantification of which may serve to predict sepsis and mortality in critically ill patients ${ }^{7}$ The endothelium becomes dysfunctional due to the sustained onslaught of the inflammatory molecules and the simultaneous oxidative stress. An early event is the loss of barrier integrity leading to systemic capillary leak. ${ }^{8}$ The glomerular manifestation of this enhanced capillary permeability is increased excretion of albumin in the urine. ${ }^{9}$ Microalbuminuria defined as $30-300 \mathrm{mg} / \mathrm{day}$ of albumin excretion in urine. It occurs rapidly after an acute inflammatory insult such as sepsis and persists in patients with complications. ${ }^{10-15}$ It is a common finding in critically ill patients where it has shown promise not only as a predictor of organ failure and vasopressor requirement but also of mortality better than APACHE II score and sequential organ function scores. ${ }^{16-21}$

\section{Objectives}


1. To evaluate the degree of microalbuminuria and correlate with severity of sepsis.

2. To evaluate levels of TNF $\alpha$ and severity of sepsis.

3. To correlate degree of microalbuminuria with levels of TNF $\alpha$ in patients with sepsis and its outcome.

\section{Materials \& Methods}

The present study was conducted on patients admitted to medicine ward and ICU staying more than 24 hrs at S.C.B Medical College, Cuttack are included in the study, during period of Sept'2011 - Sept'2012. American college of chest physician society of critical care medicine consensus reference definitions were used to delineate patients with SIRS, SEPSIS, SEVERE SEPSIS, SEPTIC SHOCK and MODS. A detailed history taking and thorough clinical examination was done in all cases. APACHE II score was calculated in all cases at the time of admission. Urine samples were collected within 6hrs of admission and again after 24 hrs.Urinary microalbumin assay by microalbumin kit (pyrogallol red immunobiometric method). Urinary creatinine estimation by creatinine kit (Mod jaffe's kinetic method).Albumin creatinine ratio (ACR) was estimated. ACR $30-299 \mathrm{mg} / \mathrm{gm}$ is microalbuminuria. $>300 \mathrm{mg} / \mathrm{gm}$ is clinical proteinuria. TNF $\alpha$ is estimated in patients with sepsis by ELISA. (bioplex PRO ${ }^{\mathrm{TM}}$ ).Patients were followed up untill discharge or death.Following cases are excluded from the study- Diabetes mellitus, Hypertension, Nephrotic syndrome, CKD (sonographic features of $\mathrm{CKD}$ and or GFR $<30 \mathrm{ML} / \mathrm{min}$ ), Cardiac failure, Immunocompromised host.

\section{Results}

The present study "Evaluation of Microalbuminuria and serum TNF $\alpha$ in patients with sepsis and their correlation with disease severity", Out of 75 cases of sepsis, 52 were males $(69.33 \%)$ and 23 were females (30.67\%). Out of total 75 cases 30 were sepsis (40\%), 20 cases of severe sepsis (26.67\%), septic shock 16 cases (21.33\%) and MODS were 9 cases (12\%). Highest mortality is in MODS (77.77\%) followed by septic shock $(43.75 \%)$,in severe sepsis mortality is also high (35\%).

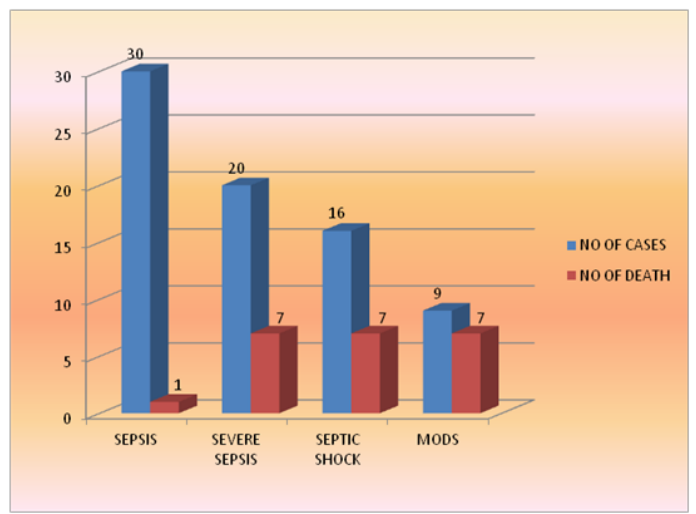

Mortality is least in sepsis (3.33\%).

APACHE II score was calculated at the time of admission of all patients with sepsis $(\mathrm{n}=75)$, it could distinguish patients who survived from those who did not $(\mathrm{p}=0.0001)$. Median APACHE II score of non survivors (16.5) was significantly higher than survivors (10.0). APACHE II SCORE at the time of admission could distinguish patients of sepsis from other categories (severe sepsis, septic shock and MODS) with a high significance $(\mathrm{p}=0.0001)$. APACHE II SCORES were highest in MODS with a median of 19, followed by septic shock (15), severe sepsis (13.5) and were least in sepsis $[$ Median $=9]$.

Comparison of ACR (Microalbumin Creatinine Ratio) between sepsis \& control cases Normalised micro-albumin creatinine ratio

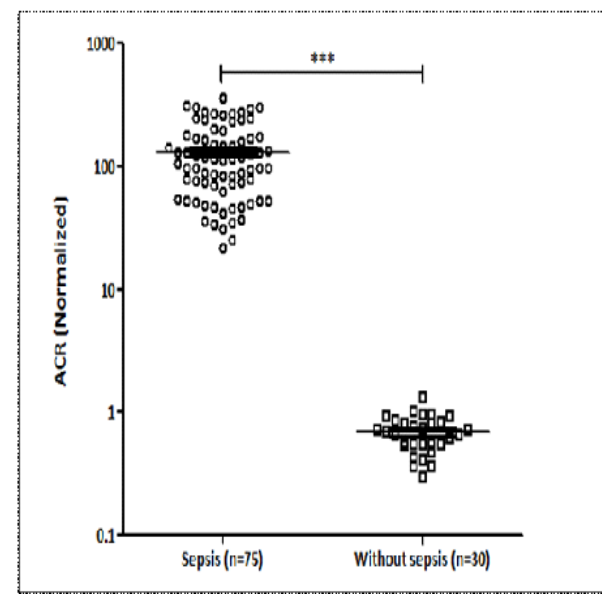
Normalised micro-albumin creatinine ratio
$(\mathrm{ACR})$ can distinguish patients with sepsis
$(\mathrm{n}=75)$ from control group $(\mathrm{n}=30) \cdot \mathrm{p}=0.0001$.
Median ACR of sepsis $(32.48 \mathrm{mg} / \mathrm{G})$ was
significantly high than control $(0.6815 \mathrm{mg} / \mathrm{G})$

(ACR) can distinguish patients with sepsis $(\mathrm{n}=75)$ from control group $(\mathrm{n}=30) . \mathrm{p}=0.0001$. Median ACR of sepsis $(32.48 \mathrm{mg} / \mathrm{G})$ was significantly high than control $(0.6815 \mathrm{mg} / \mathrm{G})$

Comparison of normalized ACR between Survivors \& Nonsurvivors 


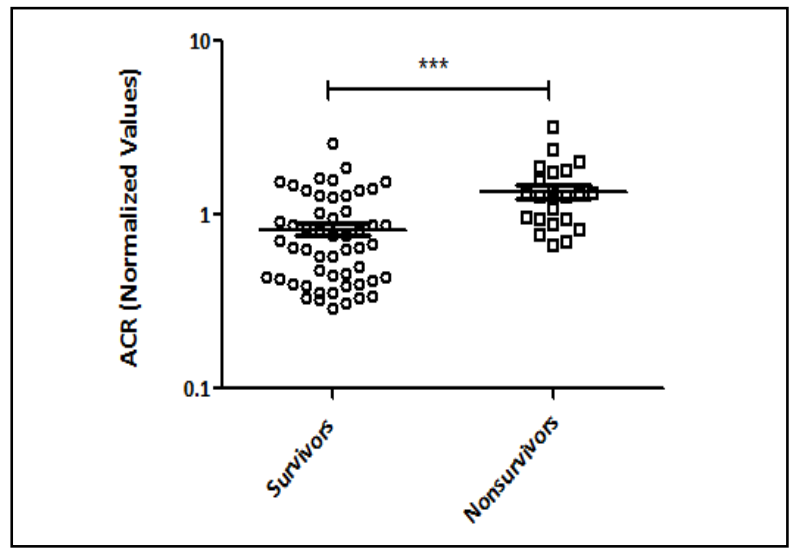

Normalised values of urinary micro-albumin creatinine ratio (ACR) could differentiate patients who survived from who did not $(\mathrm{p}<0.001)$

Comparison of normalized ACR among categories of Sepsis

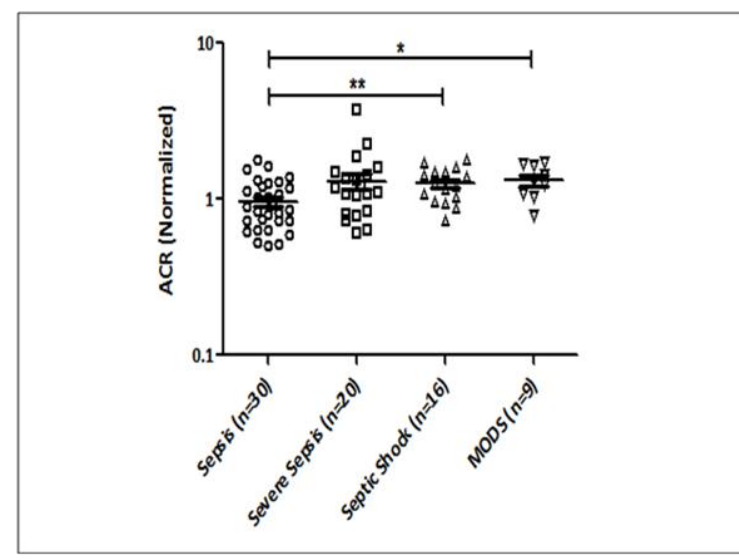

Normalised values of urinary microalbumin creatinine ratio $(\mathrm{ACR})$ can distinguish patients of sepsis from septic shock $(\mathrm{p}=0.007)$ and $\operatorname{MODS}(\mathrm{p}=0.03)$.

Comparision of TNF $\alpha$ levels between Survivors \& Nonsurvivors

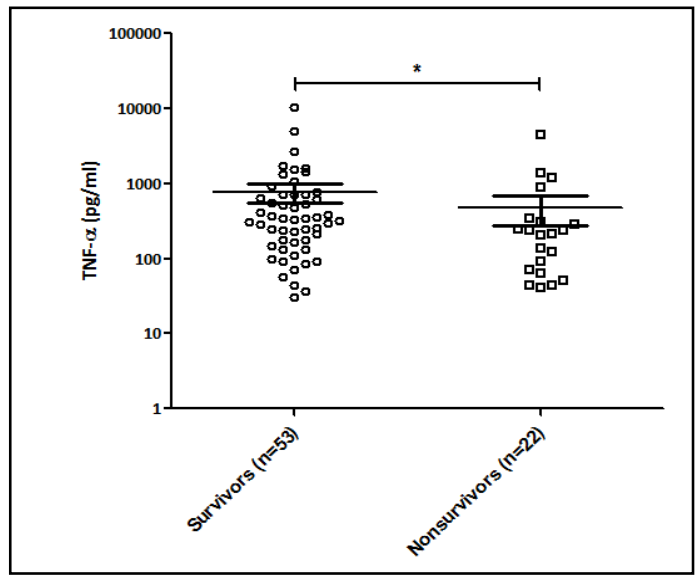

Estimated TNF- $\alpha$ values can distinguish survivors from non-survivors $(\mathrm{p}=0.04)$. Median TNF- $\alpha$ is high in survivors $(325.6 \mathrm{pg} / \mathrm{ml})$.TNF- $\alpha$ was significantly low in patients with septic shock (median=126.8) from those of sepsis (median=428.8). It can help in distinguishing patients with septic shock from patients of sepsis $(\mathrm{p}=0.02)$.

\section{Correlation between normalised ACR and TNF $\alpha$ in Sepsis patients}




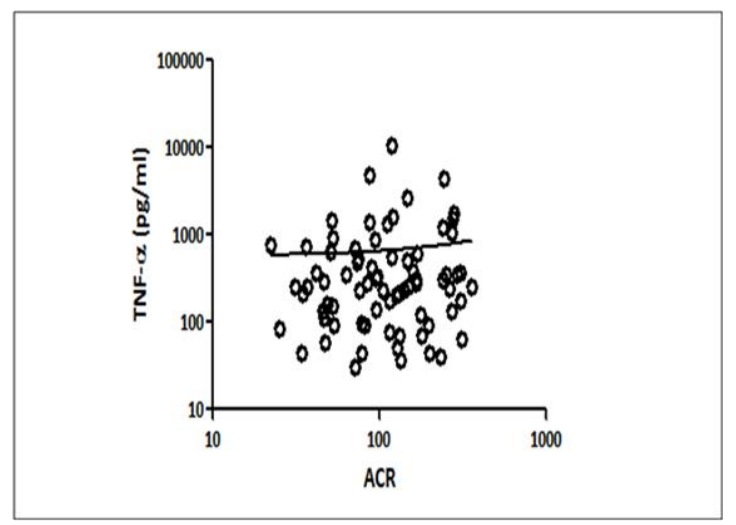

The spearman curve suggests there is no correlation between ACR (microalbumin creatinine ratio) and TNF $\alpha$ values.

\section{Discussion}

In the present study"Evaluation of Microalbuminuria and serum TNF $\alpha$ in patients with sepsis and their correlation with disease severity", out of 75 cases, 52

$(69.3 \%)$ were males and $23(30.7 \%)$ were females. 75 cases were categorised into sepsis (30), severe sepsis (20), septic shock (16) and MODS (9) . Though all age groups were involved, septic shock and MODS were common in older age group. Angus DC et al $\mathbf{( 2 0 0 1}^{1}$ reported that increasing age increases the severity of sepsis because of compromised immune system.

In this study, culture positivity was documented in 22 cases. Culture positivity according to Sands et al $(\mathbf{1 9 9 7})^{27}$ is extremely low in sepsis. Bacterial growth is found only $20-40 \%$ cases of severe sepsis and $40-70 \%$ cases of septic shock, of which $44 \%$ of cases had gram-ve infection. Gram-ve infection was predominate in our study, which corroborates with the observation of Sands et al (1997).

There were 22 deaths out of 75 cases studied. Mortality was highest in MODS. 7 out of 9 MODS (77.77\%) died. Mortality progressively decreased with less severity of disease, namely in septic shock [7 out of 16 cases $(43.75 \%)$ ], severe sepsis [7 out of 20 cases (35\%)] and least in sepsis [1 out of 30 cases (3.33\%)] (Table: 3). Mortality rates are closely associated with severity of sepsis and the number of organs involved Martin et al $(2003)^{64}$.

APACHE II (Acute Physiology and Chronic Health Evaluation II) Score was applied to distinguish categories of sepsis. The Scores were highest in MODS (median score $=19$ ), followed by septic shock, severe sepsis and least in sepsis. Knaus WA et al (1985) observed that patients admitted to intensive care unit (ICU) higher score of APACHE II within 24 hours of admission corresponded to more severe disease and higher risk of death. The scoring system is a useful, validated clinical tool to classify severity of illness and prognosticate outcome.

Urine microalbumin creatinin ratio (ACR) was assessed within 6 hours of admission and it showed a significant difference between sepsis $(\mathrm{n}=75)$ and control group $(\mathrm{n}=30),[\mathrm{p}<0.0001]$. Median ACR in sepsis $(32.48 \mathrm{mg} / \mathrm{G})$ was very significantly high compared to control group $(0.6815 \mathrm{mg} / \mathrm{G})$. Surupa Basu et al (2010) ${ }^{7}$ observed [ $\mathrm{n}=94$ ] that median ACR in sepsis patients was $(206.5 \mathrm{mg} / \mathrm{G})$, which was significantly high compared to non sepsis group $(76.4 \mathrm{mg} / \mathrm{G})[\mathrm{p}=0.0016]$. Gosling et al (2006) ${ }^{18}$ also reported that urine microalbumin changes rapidly within first 6 hours following ICU admission and could predict ICU mortality and Ionotrope requirement comparable to APACHE II Score. Our study revealed similar observations that urine microalbumin creatinine ratio (ACR1) within first 6 hours of hospital admission can be an early diagnostic marker of sepsis due to release of proinflammatory molecules leading to endothelial dysfunction and systemic capillary leak. It could also distinguish between survivors and non-survivors $(\mathrm{p}<0.05)$. ACR2, on urine sample collected after 24 hours could also predict the outcome of disease $(p<0.001)$, even better than ACR1. Normalised ACR denotes change of ACR values in day 2 with respect to day 1 . Therefore normalised values of ACR is an important parameter to differentiate survivors from non-survivors $(\mathrm{p}<0.001)$. K L Mackinoon et al $(\mathbf{2 0 0 0})^{\mathbf{1 6}}$ alsoreported thaturinary microalbumin to creatinine ratio measured within $6 \mathrm{hrs}$ of admission to ICU significantly predicted outcome $(\mathrm{p}=0.01)$ and helped in rapid identification of patients at increased risk of developing organ failure and death. Gosling et al (2006) ${ }^{9}$ had observed that urine microalbumin increased rapidly within 6 hours following ICU admission and predicted ICU mortality.

We further assessed normalised ACR as a marker to differentiate categories of sepsis. Very high ACR values were observed in septic shock and MODS and least in sepsis. Normalised values of ACR (urine microalbumin creatinine ratio) could differentiate patients of sepsis from septic shock $(\mathrm{p}=0.007)$ and MODS $(\mathrm{p}=0.03)$.Omar Abid et al (2001) ${ }^{\mathbf{1 7}}$ reported, the positive predictive value of increasing microalbuminuria (ACR) to differentiate MODS from other categories of sepsis to be 50\% and negative predictive value as $96 \%$. 
Sequential analysis of urinary microalbumin excretion could predict the onset of MODS.

Normalised ACR also showed a highly significant positive co-relation with APACHE II Score $(\mathrm{p}=0.004, \mathrm{r}=0.3276)$. K $\mathbf{L}$ Mackinnon et al $\mathbf{( 2 0 0 0 )})^{\mathbf{1 6}}$ observed in their study that Increase in urinary microalbumin excretion occured in patients with the highest APACHE II and MODS. Gosling et al (2006) ${ }^{\mathbf{2}}$ also observed the same correlation between microalbuminuria, APACHE II and SOFA in predicting ICU mortality and inotrope requirement. We assessed TNF $\alpha$ in 75 cases of sepsis and observed that TNF $\alpha$ could distinguish survivors from non survivors. In survivors $(\mathrm{n}=53) \mathrm{TNF} \alpha$ was significantly high compared to nonsurvivors $(\mathrm{n}=22)$. Richard $\mathbf{S}$ Hotchkiss et al $\mathbf{( 2 0 0 3}^{4}$ observed the initial response in sepsis is hyper inflammatory but the response rapidly progresses to hypoinflammatory state. The role of TNF $\alpha$ in combating infection has recently been underscored. Reversal of Th1 to Th2, a hypoinflammatory response, improves survival among patients with septic shock.

Richard S Hotchkiss et al (2003) ${ }^{4}$ in their study showed that measurement of circulating concentrations of inflammatory mediators was useful in evaluating the stages of sepsis and preventing the administration of anti-inflammatory agents as a treatment. Although cytokines are considered to be culprits, they have beneficial effects too. OpalSM et al (2000) ${ }^{46}$ observed although high concentrations of both pro and anti inflammatory molecules were found in patients with septic shock, the mediator balance seems to be antiinflammatory. Eskandari MK et al (1992) ${ }^{\mathbf{3 8}}$ observed that Anti TNF anti body therapy in septic shock failed to prevent endo-toxemia. In the present study TNF $\alpha$ could differentiate patients of septic shock from sepsis. TNF $\alpha$ was significantly low in patients of septic shock $(\mathrm{p}=0.02)$.

Our study demonstrated that TNF $\alpha$ is high in all patients of sepsis, but it came down gradually as the patient progressed to septic shock. This may be due to the initial hyperinflammatory response of sepsis rapidly progressing to hypoinflammatory state, where proinflammatory cytokine levels (Th1 response) fall and anti inflammatory cytokines (Th2 response) rise; Richard S Hotchkiss et al (2003) ${ }^{4}$. In the hypoinflammatory phase administration of anti TNF $\alpha$ agents failed to improve the outcome of the patients (Eskandari MK et al, 1992) ${ }^{38}$. Though both TNF $\alpha$ and urine microalbumincreatinine ratio (ACR) individually could predict outcome of patients and categorise patients of sepsis but there was no significant correlation between them (Figure:14). According to Richard S Hotchkiss et al (2003) ${ }^{4}$; the individual response to SIRS is determined by many factors, including the virulence of the organism, the size of the inoculum, and the patient's coexisting conditions, age, and polymorphisms in genes for cytokines. Similarly Freedman et al (2000) ${ }^{67}$ opined that, the reasons for the failure to detect high TNF- $\alpha$ or IL-1 in the circulation of some patients of sepsis are multiple. The production of TNF- $\alpha$ and IL-1 levels is episodic. Macrophages exposed to continuous levels of endotoxin, release TNF- $\alpha$ and IL-1 in a burst over several hours and then become tolerant to repeated or continued exposure. This development of tachyphylaxis remains a hallmark cytokine response to exposure to bacterial cell products. Another possible reason for failing to detect TNF- $\alpha$ and IL-1, is that the plasma halflives of TNF- $\alpha$ and IL-1 are generally less than 60 minutes. Since the study was cross sectional with single point of collection of plasma, the estimated value of TNF $\alpha$ may not correlate with ACR. Since sepsis is a dynamic process, a time sequential study could have helped to explain this lack of correlation.

\section{Conclusion}

Sepsis is the culmination of complex interactions between the infecting microorganism, the host inflammatory and immune responses. Initial response is hyperinflammatory but rapidly changes to hypoinflammatory. The rationale of therapeutic targets in sepsis has arisen from concepts of pathogenesis. Both the host responses and the characteristics of the infecting organism influence the outcome of sepsis.

APACHE II scoring system is a useful, validated clinical tool to classify severity of illness and prognosticate outcome. Urine microalbumin, generally expressed as the urinary albumin to creatinine ratio(ACR)to correct variations in urinary flow rate and concentration, may be an early diagnostic marker of sepsis due to release of proinflammatory molecules (eg;TNF $\alpha$ ) leading to endothelial dysfunction and capillary leak.

Urine microalbumin(ACR) increases rapidly in sepsis. Trends of microalbuminuria not only correlates severity of sepsis but also predicts mortaliy, inotrope requirement and impending organ failure in sepsis equivalent to time tested APACHE II Score.

Sepsis being a dynamic process, a time sequential study may be useful in explaining correlation of cytokine profile (TNF $\alpha)$ with values of microalbuminuria. 


\section{References}

[1] Agnus DC, Linde-Zwirble WT, LidickerJ.Epidemiology of severe sepsis in the United States: Analysis of incidence, outcome and associated costs of care. Crit Care Med 2001;29: 1303-10.

[2] Todi S, Chatterjee S, Bhattacharyya M. Epidemiology of severe sepsis in India. Crit Care 2007;11:65.

[3] Rivers E, Nguyen B. Havstad S, Ressler J, Muzzin A, Knoblieh B. et al. Early goal directed therapy in the treatment of severe sepsis and septic shock. N Engl J Med 2001;345:1368-77.

[4] Hotchkiss RS, Karl IE. The pathophysiology and treatment of sepsis. N Engl J Med 2003,348;138-50.

[5] Robert S Munford, severe sepsis and septic shock, Harrisons Internal Medicine $18^{\text {th }}$ Edition, $271 ; 2223-2232$.

[6] ArteriosclerThrombVasc Biol. 2007 Jun;27(6):1269-75

[7] S Basu, M Bhattacharya, T K Chatterjee, S Choudhuri, S Todi, AMajumdar.Microalbuminuria a novel biomarker of sepsis. Indian $\mathrm{j}$ crit care med 2010; vol 14 issue 1.

[8] Aird William C. The role of endothelium in severe sepsis and multiple organ dysfunction syndrome. Blood 2003;101:3765-77.

[9] Gosling P Microalbuminuria: A marker of systemic disease. Br J Hosp Med 1995;54:285-90.

[10] Berton G Citro T, Palmieri R, Petneco S. De Toni R, Palatini P, Albumin excretion rate increases during acute myocardial infarction and strongly predicts early mortality. Circulation 1997;96:3338-45.

[11] De Gaudio AR, Spina R, Di Filippo A, Feri M. Glomercular permeability and trauma: A correlation between microalbuminuria and injury severity score. Crit Care Med 1999;27:2105-8.

[12] De Gaudio AR, Adembric C, Grechi S, Novelli GP. Microalbuminuria as an early index of impairment of glomerular permeability in postoperative septic patients. Intensive Care Med 2002;26:1364-8.

[13] Szakmany T, Molnar Z. Increased glomerular permeability and pulmonary dysfunction following major surgery: Correlation of microalbuminuria and $\mathrm{PaO2} / \mathrm{Fi02}$ ratio. AetaAnaesthesiolScand 2004;48:704-10.

[14] Yew WS, Pal SK. Correlation of microalbuminuria and outcome in patients with extensive burns. Br. J Anaesth 2006;97:499-502

[15] Terao Y, Takada M. Tanabe T, Ando Y, Fukusaki M. Sumikawa K. Microalbuminuria is a prognostic predictor in aneurysmal subarachnoid hemorrhage. Intensive Care Med 2007;33:1000-6.

[16] MacKinnon KL, Molnar Z, Lowe D, Watson ID, Shearer E. Use of microalbuminuria as a predictor of outcome in critically ill patients. Br. J Anaesth 2000;84:239-41.

[17] Abid O, Sun Q, Sugimoto K, Merean D, Vincent JL. Predictive value of microalbuminuria in medical ICU patients: Results of a pilot study. Chest 2001; 120:1984-8.

[18] Gosling P, Brudney S. McGrath L, Riseboro S, Manji M. Mortality prediction at admission to intensive care: A comparision of microalbuminuria with acute physiology scores after 24 hours. Crit Care Med 2003; 31:98-103.

[19] Thorevska N, Sabahi R, Upadya A, Manthous .Microalbuminuria in critically ill medical patients: Prevalence, predictors, and prognostic significance. Crit Care Med 2003;31:107581.

[20] Gosling P, Czyz J, Nightingale P, Manji M. Microalbuminuria in the intensive care unit: Clinical correlates and association with outcomes in 431 patients. Crit Care Med 2006;34:2158-66.

[21] Goral S, Carr B, Nelson P: Does microalbuminuria predict illness severity in critically ill patients on the intensive care unit? A systematic review. Crit Care Med 2006;34:1805-10.

[22] Weimar sepsis Update:SepsisHistory,German sepsis society $5^{\text {th }}$ International congress sept 2011

[23] Namas, Ruben Zamora, Rajaienamas, GaryAn: Sepsis; Somethingold, somethingnew, and a systems view. Journal of critical care 2011.05.025.

[24] Davies A, Green C, Hutton J. Severe sepsis: a European estimate of the burden of disease in ICU. Int Care Med 2001;27:S284.

[25] James A Russel: Management of sepsis. N Engl J Med 2006, 355; 1699713.

[26] Todi S; Sepsis: Newhorizons. Indian J of Ctit Care Med January - March'2010 Vol. 14 issue 1. 